\title{
Response to: Clinical Use, Quality of Life and Cost-Effectiveness of Spinal Cord Stimulation Used to Treat Failed Back Surgery Syndrome
}

\author{
Przemyslaw Maciej Waszak ${ }^{1,2}$, Marta Modrić ${ }^{3}$, Agnieszka Paturej ${ }^{4}$, \\ Stanislav M. Malyshev ${ }^{5}$, Agnieszka Przygocka ${ }^{6}$, Hanna Garnier ${ }^{7}$, Tomasz Szmuda ${ }^{8}$ \\ ${ }^{1} J a n u s z$ Korczak Memorial Voivodeship Hospital, Stupsk, Poland \\ ${ }^{2}$ Department of Hygiene and Epidemiology, Medical University of Gdansk, Gdansk, Poland \\ ${ }^{3}$ Varaždin General Hospital, Varaždin, Croatia \\ ${ }^{4}$ Students' Scientific Association, Department of Neurosurgery, Medical University of Gdansk, Gdansk, Poland \\ ${ }^{5}$ Federal Almazov North-West Medical Research Centre, St. Petersburg, Russia \\ ${ }^{6}$ Poznań University of Medical Sciences, Poznań, Poland \\ ${ }^{7}$ Medical University of Gdansk, Gdansk, Poland \\ ${ }^{8}$ Department of Neurosurgery, Medical University of Gdansk, Gdansk, Poland
}

Dear Editor,

We appreciate the letter regarding the review on spinal cord stimulation (SCS) in failed back surgery syndrome (FBSS) [1]. We thank the authors who carefully read our manuscript and provided valuable comments. Because this review took under consideration evidence for clinical utility, quality of life, and cost-effectiveness, our cautious conclusions were based on all mentioned factors.

We regret that the methodology section was unclear and could be misleading for some readers. Our work was not a systematic review (although we did use preferred reporting items for systematic reviews and meta-analyses) article selection guidelines [2]); therefore, we have not included citations for all papers found in medical databases (>70 studies). The authors of the letter recalled research conducted by Zucco et al. [3], which was known to us while drafting the manuscript. This Italian research is an observational study whose results we await. The aim of this trial was to evaluate patients' quality of life and costeffectiveness in real-world healthcare settings. The authors recruited 80 patients with FBSS from multiple hospitals with 24 months of follow-up. They found significant improvement in patients' quality of life as well as accepted cost-effectiveness, when willingness to pay was set at $€ 60,000$ per year.

Despite several good methodologically studies (including the study conducted by Zucco et al. [3]), the evidence for SCS in FBSS in reliable long-term data remains insufficient. Due to FBSS chronic nature, prolonged observations could be particularly important. If continued, this study may provide another valuable conclusion.

We agree that SCS can be both of clinical use and costeffective, but there are several limitations regarding this technique which was discussed further in our review.

Received May 29, 2017; Accepted May 29, 2017

Corresponding author: Przemyslaw Maciej Waszak

Janusz Korczak Memorial Voivodeship Hospital in Słupsk, ul. Prof. Lotha 26; 76-200 Słupsk, Poland

Tel: +48-663-534-485, E-mail: p.waszak@gumed.edu.pl 


\section{Conflict of Interest}

No potential conflict of interest relevant to this article was reported.

\section{References}

1. Waszak PM, Modric M, Paturej A, et al. Spinal cord stimulation in failed back surgery syndrome: review of clinical use, quality of life and cost-effectiveness. Asian Spine J 2016;10:1195-204.
2. Liberati A, Altman DG, Tetzlaff J, et al. The PRISMA statement for reporting systematic reviews and metaanalyses of studies that evaluate health care interventions: explanation and elaboration. J Clin Epidemiol 2009;62:e1-34.

3. Zucco F, Ciampichini R, Lavano A, et al. Cost-effectiveness and cost-utility analysis of spinal cord stimulation in patients with failed back surgery syndrome: results from the PRECISE Study. Neuromodulation 2015;18:266-76. 